DOI: 10.1515/awutp -2015-0207

\title{
A SOLID STATE PYRANOMETER
}

\author{
Anca Laura Dumitrescu, Marius Paulescu, Aurel Ercuta
}

Faculty of Physics, West University of Timisoara, RO-300223, Timisoara, Romania

\begin{abstract}
Article Info
Received: 13.09.2015

Accepted: 11.11.2015
\end{abstract}

\section{Keywords:}

Pyranometer, diode $1 N 4148$

\begin{abstract}
The construction of a solid state device-based pyranometer designated to broadband irradiance measurements is presented in this paper. The device is built on the physical basis that the temperature difference between two bodies of identical shape and external surface area, identically exposed to the incident radiation, but having different absorption and heat transfer coefficients (e.g. one body is painted white and the other is painted black), is proportional to the incident irradiance. This proportionality may be put in evidence if the two bodies consisting of identical arrays of correspondingly painted semiconductor diodes, due to the thermal behaviour of their $p$ - $n$ junction. It is theoretically predicted and experimentally confirmed that the voltage drop across a diode passed through a constant forward current linearly decreases with the temperature of the junction. In other words, a signal proportional to the irradiance of the light source may be obtained via conventional analog electronics. The calibration of the apparatus, as performed by means of a professional device (LP PYRA 03), indicates a good linearity.
\end{abstract}

\section{The principle of the method}

Consider first a solid body exposed to electromagnetic radiation (Fig.1) emitted by a source (e.g. an electric bulb) of spectral distribution close to that of a black body when heated to a sufficiently high temperature.

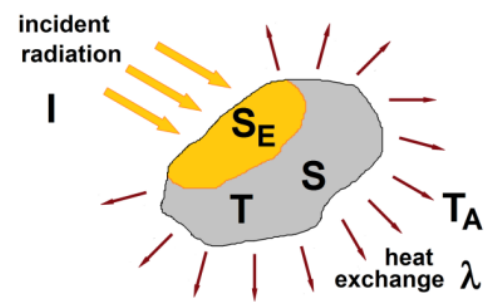

Fig. 1. A solid body partially exposed to electromagnetic radiation (light)

Let:

$I\left[\mathrm{~W} / \mathrm{m}^{2}\right]$ - the irradiance of the source (assumed a point source),

$A$ - the absorption coefficient of the body surface 
$S$ - the area of the external surface

$S_{E}$ - the area of the surface exposed to radiation

$C$ - the heat capacity of the body

$\lambda$ - the coefficient of heat exchange between the body and the environment

$T$ - the temperature of the body

$T_{\mathrm{A}}$ - the environmental temperature

In these terms, the energetic balance is expressed as:

$$
A I S_{E} d t=C d T+\lambda S\left(T-T_{A}\right) d t
$$

which is a first order ODE (ordinary differential equation), of solution:

$$
T(t)=T_{A}+\frac{S_{E} A}{S \lambda}\left[1-\exp \left(-\frac{S \lambda}{C} t\right)\right] I
$$

with the asymptotic form (the equilibrium temperature):

$$
T_{e}=\lim _{t \rightarrow \infty} T(t)=T_{A}+\frac{S_{E} A}{S \lambda} I
$$

Consider now a pair of identical bodies (having the same values of $\mathrm{S}$ and $\mathrm{C}$ ), except for the fact that one is painted white and the other is painted black. Let:

$A_{W}, A_{B}$ - the absorption coefficients of the two bodies (white and black, respectively)

$S_{W}, S_{B}$ - the area of the external surface of the two bodies

$\lambda_{W}, \lambda_{B}$ - the coefficients of heat exchange between the white and black bodies and the ambiental athmosphere

$T_{W}, T_{B}$ - the temperatures of the two bodies

The two bodies are identically exposed to radiation (and thus $S_{E W}=S_{E B}=S_{E}$ the areas of the surface exposed to radiation); see Fig.2 in this sense.
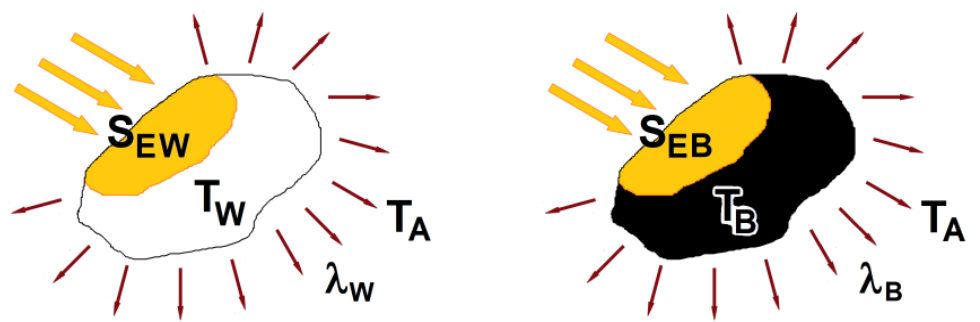

Fig. 2. A white and a black solid bodies partially exposed to electromagnetic radiation (light) 
Thus, the difference between the equilibrium temperatures of the two bodies will be

$$
\Delta T_{e}=T_{e B}-T_{e W}=\frac{S_{E}}{S}\left(\frac{A_{B}}{\lambda_{B}}-\frac{A_{W}}{\lambda_{W}}\right) I
$$

In other words, $\Delta T_{e}$ is a direct measure of the irradiance $I$ of the light source.

Consider now that the white and black bodies are two silicon diodes, one painted white and the other painted black. As pointed out previously, the electric behaviour of the ideal diode is well approximated by the modified Shockley equation:

$$
j_{D}=j_{S}\left[\exp \left(-\frac{e U_{D}}{m k_{B} T}\right)-1\right]
$$

which describes the dependence of the forward current density $j_{D}$ on the applied polarization voltage $U_{D}$; here, $e=1.6 \times 10^{-19} \mathrm{C}$ is the elementary charge and $j_{s}$ is the saturation current density. If the electron-hole recombination process is taken into account, the numeric factor $m$ at the denominator of the exponent takes values close to 2 for silicon; an example in this sense is shown in Fig. 3 for the popular Si diode 1N4148, where a least squares fitting based on Eq. (5) yields $m=2.03$. The well-known expression of the saturation current density is [1]:

$$
j_{s}=e n_{i}^{2}\left[\frac{D_{n}}{L_{n} N_{A}}+\frac{D_{p}}{L_{p} N_{D}}\right]
$$

where $D_{n}, D_{p}$ and $L_{n}, L_{p}$ are the diffusion coefficients and the diffusion lengths for electrons and holes, respectively, while $N_{A}$ and $N_{D}$ are the concentrations of the acceptor and donor doping atoms. Then, if we write the intrinsic concentration of the carriers as:

$$
n_{i}=2\left[\frac{2 \pi k_{B} T}{h^{2}}\right]^{\frac{3}{2}}\left(m_{n}^{*} m_{p}^{*}\right)^{\frac{3}{4}} \exp \left(-\frac{E_{g}}{k_{B} T}\right)
$$

where $m_{n}^{*}$ and $m_{p}^{*}$ are the effective masses of the electrons and holes, respectively, $h$ is the Planck's constant, and if we take into account the temperature dependences of ratios:

$$
\frac{D_{n}}{L_{n}}=\left[\frac{k_{B} T}{e} \cdot \frac{\mu_{n}}{\tau_{n}}\right]^{\frac{1}{2}}=a_{n} T^{\frac{1}{2}} ; \frac{D_{p}}{L_{p}}=\left[\frac{k_{B} T}{e} \cdot \frac{\mu_{p}}{\tau_{p}}\right]^{\frac{1}{2}}=a_{p} T^{\frac{1}{2}}
$$


where $\mu_{n}, \mu_{p}$ and $\tau_{n}, \tau_{p}$ are the carriers mobilities and lifetimes respectively, simple calculations lead to the following expression results for the forward current:

$$
I_{D}=\text { Const } \times T^{7 / 2} \exp \left(-\frac{E_{g}}{k_{B} T}\right)\left[\exp \left(\frac{e U_{D}}{\mu k_{B} T}\right)-1\right]
$$

where Const embeds all the constant quantities (including the $p-n$ junction area). It is easy to observe that for measurable values of $I_{D}$ the voltage $U_{D}$ across the diode should exceed a reasonable value (say, $0.4 \mathrm{~V}$ ); accordingly,

$$
\exp \left(\frac{e U_{D}}{\mu k_{B} T}\right)>>1
$$

(e.g. the value is 2345 for $U_{D}=0.4 \mathrm{~V}$ in our case) and thus unity may be neglected in the squared

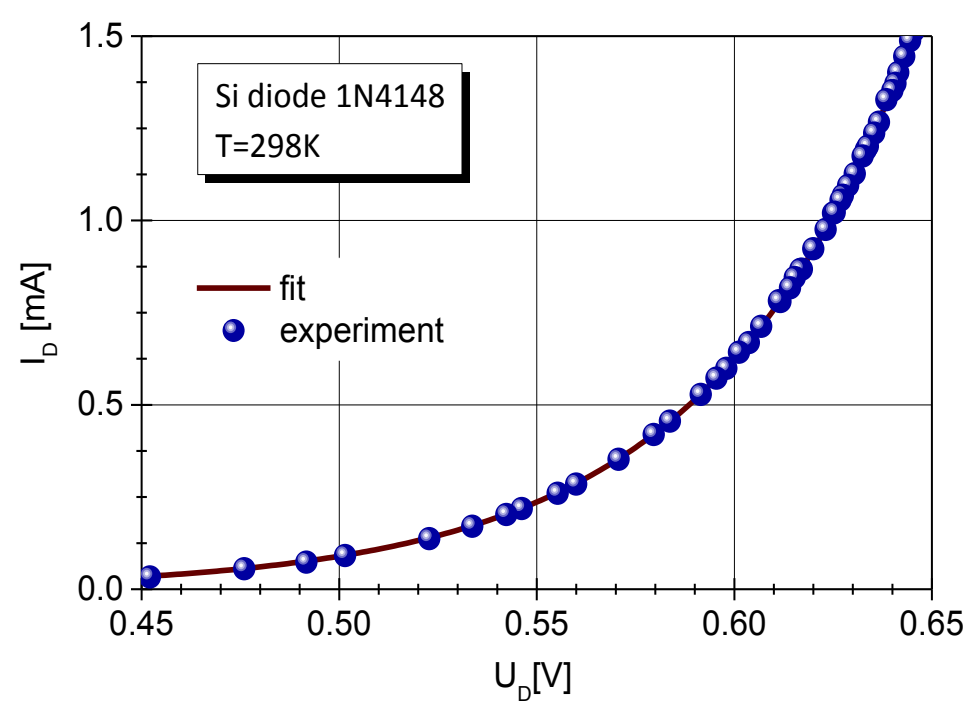

Fig. 3. The voltage-current characteristic of the silicon diode $1 \mathrm{~N} 4148$ at room temperature

brackets in the right-hand term of Eq. (9). Accordingly this may be rewritten as:

$$
I_{D}=\text { Const } \times T^{7 / 2} \exp \left(\frac{e U_{D}}{\mu k_{B} T}-\frac{E_{g}}{k_{B} T}\right)
$$

and, from here, as:

$$
\ln (\text { Const })+\frac{7}{2} \ln (T)+\frac{e U_{D}}{\mu k_{B} T}-\frac{E_{g}}{k_{B} T}=\ln \left(I_{D}\right)
$$

We shall note here that the energy gap $E_{g}$ slightly decreases with increasing temperature, following a quasilinear dependence of the form: 


$$
E_{g}(T)=E_{g 0}-\alpha_{g} T
$$

with $\alpha_{g} \approx 1.3 \times 10^{-4} \mathrm{eVK}^{-1}$ for Si. By dividing $E_{g}$ by $e$, we may write:

$$
V_{g}(T)=V_{g 0}-\alpha_{g}^{*} T
$$

with $\alpha_{g}^{*} \approx 1.3 \times 10^{-4} V K^{-1}$. Accordingly, Eq. (12) may be rewritten as:

$$
U_{D}(T)=\mu V_{g 0}-\left[\mu\left(\alpha_{g}^{*}+\frac{7}{2} \frac{k_{B}}{e} \ln (T)\right)-\ln \left(I_{D}\right)+\ln (\text { Const })\right] T
$$

Let us note that for the temperature range of interest in our application, say between $25^{\circ} \mathrm{C}$ and $50^{\circ} \mathrm{C}$, the first term in the square brackets in the right-hand side of Eq. (15) is constant within $1 \%$ precision, i.e. $1.85 \times 10^{-3}<\alpha_{g}^{*}+(7 / 2)\left(k_{B} / e\right) \ln (T)<1.87 \times 10^{-3}$. As a consequence, a linear decrease with temperature:

$$
U_{D}(T)=U_{D 0}-\gamma\left(T-T_{0}\right)
$$

is expected for $U_{D}$ in the above thermal range of interest (as the experiment shows $\Delta T_{e}$, is not expected to exceed $10 \mathrm{~K}$ ); here, $T_{0}$ is a reference temperature, and $U_{D 0}=U\left(T_{0}\right)$. As Figure 4 (where the experimental points are fitted with straight lines) shows, the simplified form (16) is well confirmed experimentally in the case of the $1 N 4148$ diode.

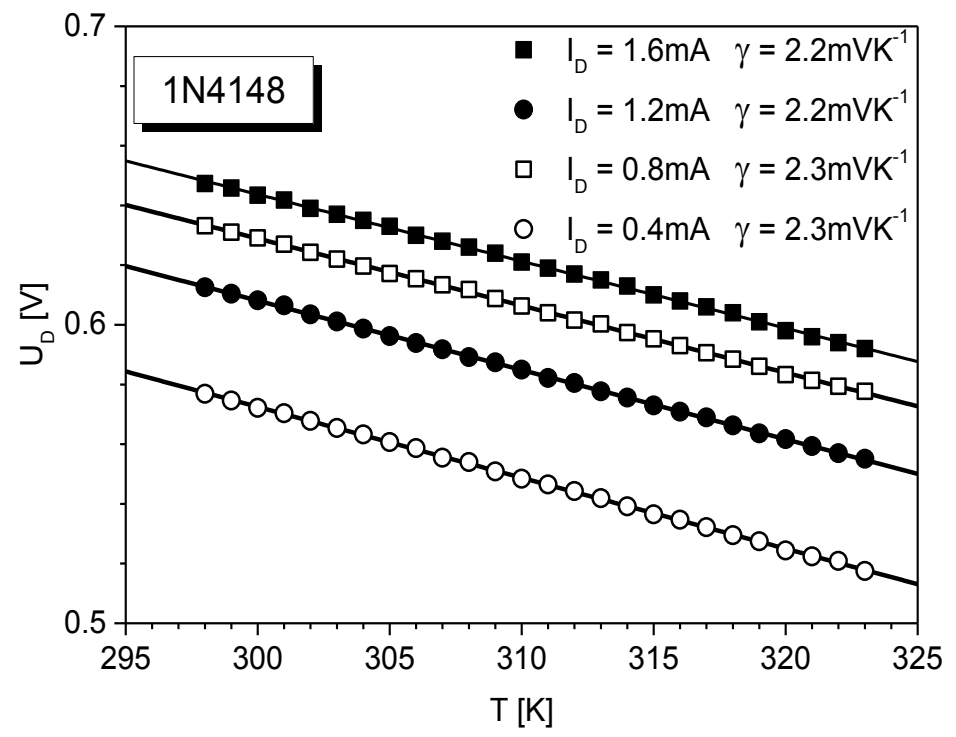

Fig. 4. The themperature dependence of $U_{D}$ at constant $I_{D}$ of the silicon diode $1 N 4148$ 
Consider then two identical arrays of $N$ diodes connected in series and passed through by a constant forward current; in one set the diodes are painted white and in the other are painted black. If the two sets of diodes are identically exposed to the electromagnetic radiation generated by a light source of irradiance $I$, at thermal equilibrium $\left(T=T_{e}\right)$ the difference

$$
U_{W B}\left(T_{e}\right)=U_{W}\left(T_{e}\right)-U_{B}\left(T_{e}\right)
$$

where

$$
U_{W}(T)=N U_{D}(T) \quad(\text { painted white })
$$

and

$$
U_{B}(T)=N U_{D}(T) \quad(\text { painted black })
$$

Combining equations (3.4), (3.18) and (3.19), one finds:

$$
U_{W B}\left(T_{e}\right)=N \alpha \gamma I
$$

The above result leads to the physical principle of an electronic device (pyranometer) by means of which the irradiance of the Sun.

\section{The apparatus (pyranometer)}

The apparatus [2] consists of an electronic circuit (Figure 5) to which two arrays of white- and black-painted silicon diodes are connected. These diodes are placed alternately in order to cover a horizontal circular area protected by a transparent shell (e.g. a glass cupole, a plastic foil, etc.), which has the main role of preventing air flow penetration.

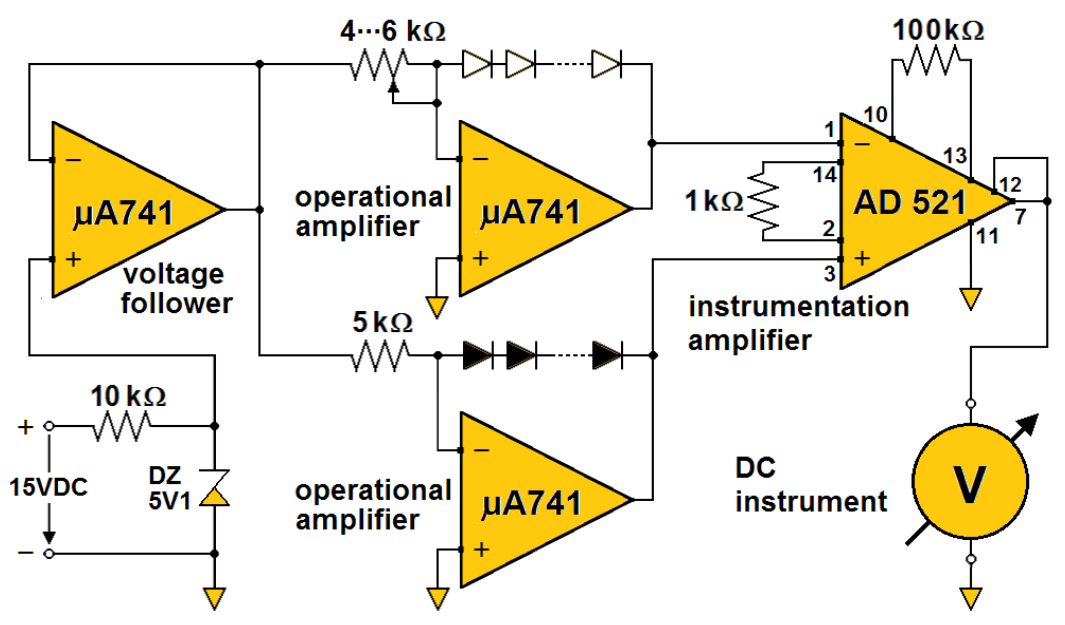

Fig. 5. The electric diagram of the pyranometer (voltage supplies and other manufacturer-recommended auxiliary circuits not shown) 
The constant current through the two sets of white-painted and black-painted diodes is ensured by connecting the diodes in series in the negative feedback loop of two operational amplifiers in inverting amplifier configuration (black and white separately), working as voltage-to-current converters: in this view, the inputs are biased with the same voltage provided by a voltage follower which repeats the reference voltage from a 5.1 Zener diode (it is known that the temperature coefficient of the Zener diodes voltage stabilization changes sign from negative to positive around $5 \mathrm{~V}$ ). For cancellation of the output offset voltage, a simple but practical solution was adopted: instead of using an additional DC voltage correction circuit, the current through the set of white-painted diodes will be slightly modified by using a rheostat $(4 \mathrm{k} \Omega . .6 \mathrm{k} \Omega)$ instead of a resistor of fixed value $(5 \mathrm{k} \Omega)$. Indeed, as figure 4 suggests, the output voltage of the voltage-to-current converter (the sum of the forward voltages across the series-connected white-painted diodes) may conveniently be changed upward or downward by slightly changing the input resistance, and thus the constant current through the diodes. Finally, the output signals from the two voltage-to-current converters are sent to the inputs of the instrumentation amplifier (of gain $100 \times$ ). The voltage follower and the two voltage-to-current converters are made using the popular industrystandard $\mu A 741$, while the instrumentation amplifier is the widely used AD521. Figure 6a shows a photo of the prototype device.

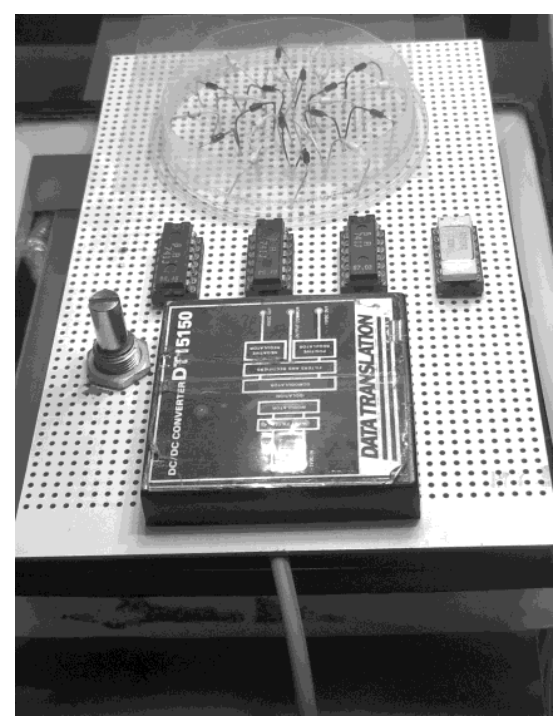

a.

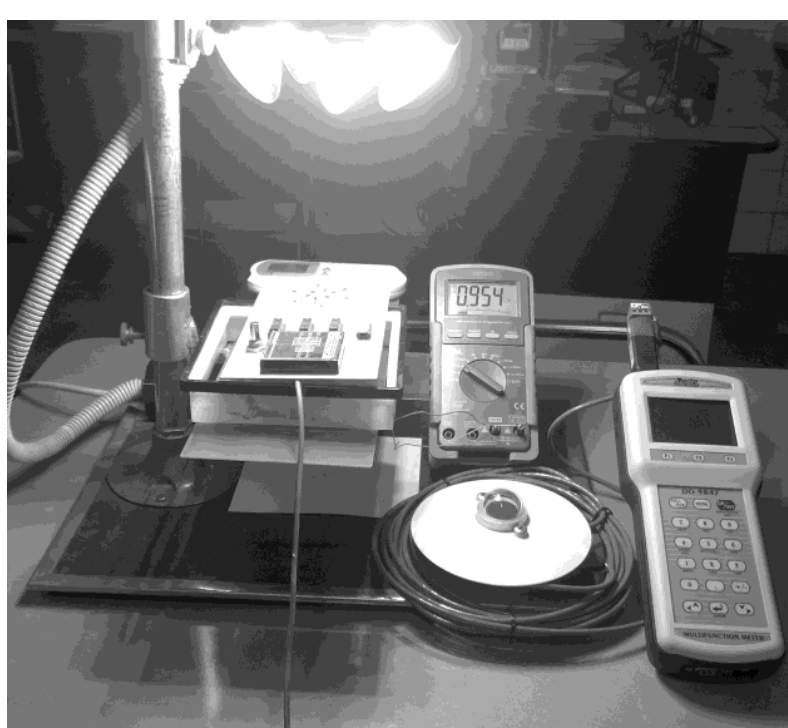

b.

Fig. 6. (a) The pyranometer and (b) The experimental stand used for calibrating the pyranometer 


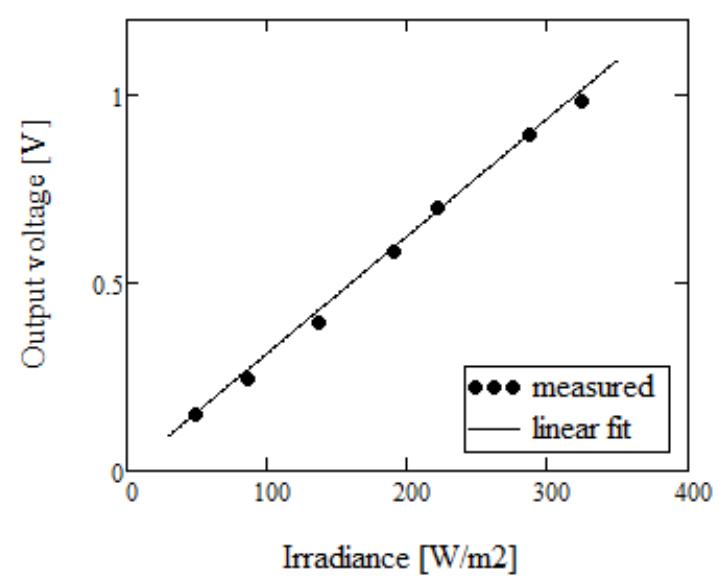

Fig. 7. Pyranometer output voltage as function of the incoming energy flux density

Calibration of the prototype device was done by means of a professional pyranometer DeltaOHM LP PYRA 03 as a reference instrument. The light source has consisted of seven bulb lamps. A photo of the experimental stand is presented in Fig. 6b. The calibration results are displayed in Fig 7. Visual inspection shows a very nice linear response of the prototype. Thus, we can simple calculate the instrument's constant $\alpha$, defined as the ratio of the output voltage to the incoming irradiance, $\alpha$ is numerically equal to the slope of the line in Fig. 7:

$$
\alpha=3.12 \cdot 10^{-3} \mathrm{VW}^{-1} \mathrm{~m}^{2}
$$

\section{Conclusions}

The black and white pyranometer proposed in this paper represents an efficient lowcost solution for measuring solar irradiance. The theoretical analysis and the laboratory calibration demonstrate that the instrument has a very good linearity.

\section{References}

[1] Sah CT, Fundamentals of solid state electronics, World Scientific, Singapore (1991).

[2] Dumitrescu AL. MSc. Dissertation, West University of Timisoara (2014). 